\title{
Filmpädagogik
}

Zurück zur Übersicht über den

Themenschwerpunkt

\section{Handlungsorientiertes Video im Fremdsprachenunterricht}

\section{MARION NIEHOFF}

\section{Einleitung}

Im Kontext des Fremdsprachenunterrichts erfahren audiovisuelle Medien traditionell groBe Aufmerksamkeit. Die ersten audiovisuellen Lehrwerke für den Fremdsprachenunterricht (FSU) wurden vor mehr als fünfzig Jahren am Institut Credif in Frankreich entwickelt (historische Übersicht in Fuß 1992, S. 155). Die technologischen Entwicklungen der folgenden Jahrzehnte und der besonders in den siebziger und achtziger Jahren des letzten Jahrhunderts rege fremdsprachendidaktische Diskurs zum Medium Film haben seitdem zu weitreichenden Entwicklungen im Umgang mit diesem Medium im FSU geführt. Neben einer Position des rezeptiven Medieneinsatzes aus dem Bereich der Unterrichtstechnologie und einer medienpädagogischen Position des aktiven Medieneinsatzes (Eggers 1981, S. 77) sind kritische Konzepte für den Umgang mit den Medien Film und Fernsehen im FSU entstanden (z. B. Röllecke 1991), die ausgehend vom Medienalltag der Lernenden zu einer besseren Kenntnis der Wirkungsmechanismen und zu einem bewussten Umgang mit Medien führen möchten. Diese verschiedenen Positionen dokumentieren auch die große Spannbreite der Einsatzmöglichkeiten für das Medium Film im Kontext des Lehrens und Lernens fremder Sprachen. Mögliche Einsatzbereiche umfassen z. B. das Lernziel Hör(seh)verstehen, die Verwendung von Filmausschnitten als Sprechanlass, die Nutzung von Video zur Schulung von Phonetik und Aussprache, den Einsatz von Literaturverfilmungen im Literaturunterricht, die Vermittlung landeskundlicher Kenntnisse oder die Nutzung von Video für den Bereich Fachsprache.

Der vorliegende Beitrag konzentriert sich auf den handlungorientierten Gebrauch der Videokamera im FSU. Er legt erprobte Vorschläge für handlungsorientiertes Video im Kontext des Lehrens und Lernens fremder Sprachen vor: zum einen für den Einsatz im Regelunterricht, zum anderen für Eigenproduktionen der Lernenden in Projektphasen. Damit wird insbesondere der Bereich der Produktion von Film im FSU thematisiert, wobei davon ausgegangen wird, dass die Rezeption und Produkti- on von Film in einer Wechselwirkung stehen und übergreifendes Ziel beider Verwendungsweisen, die Entwicklung fremdsprachiger Medienkompetenz sein sollte.

Filmpädagogisches Arbeiten und fremdsprachige Medienkompetenz

Für die Entwicklung von (fremdsprachiger) Medienkompetenz als übergeordnetes Lernziel der (handlungsorientierten) Arbeit mit Film liegen Ansätze aus der Fremdsprachendidaktik vor (z. B. Alrø 1986, Schwerdtfeger 1989, Röllecke 1991, Lindhorst u. Röllecke 1996, Kühn 1998).

Integraler Bestandteil der Verwendung von Film im FSU sollte die Entwicklung einer fremdsprachigen Medienkompetenz sein: „[...], was meint, audiovisuelle Medientexte einerseits analysieren und einschätzen zu können, sie andererseits nach eigenen Bedürfnissen zu nutzen." (Röllecke 1991, S. 406) Für den FSU bedeutet dies, das individuelle Mediennutzungsverhalten $\mathrm{zu}$ thematisieren, die alltäglichen Erfahrungen mit Medien (z. B. Vorlieben und Abneigungen) als bedeutungsvoll anzusehen, differierende Rezeptionsweisen von Filmen ernst zu nehmen und zu diskutieren (ebd., S. 407). Die handlungsorientierte Verwendung von Film zielt demnach immer auch darauf $a b$, die Analysefähigkeit und das Reflexionsvermögen der Lernenden im Hinblick auf (fremdsprachige) Medienprodukte zu entwickeln. Dieses Lehr-/Lernziel kann als Groblernziel bezeichnet werden, das nur im Verlauf eines längeren Prozess erworben werden kann. (vgl. Alrø 1987, S. 32)

Der handlungsorientierte Gebrauch der Videokamera im Regelunterricht

Der handelnde Gebrauch des Medium Films durch Lernende im FSU fördert u. a. kreative und selbst bestimmte Formen der Textproduktion und einen kritischen Umgang mit Medienprodukten. Ein vorrangiges Ziel der Filmproduktion im handlungsorientierten FSU ist es, kommunikative Funktionen verbaler und non-verbaler Mittel der Fremdsprache kennen zu lernen, anzuwenden und unter kontrastiven Gesichtspunk- 
ten $\mathrm{zu}$ analysieren. Um dieses Ziel zu erreichen, ist neben der Vorbereitung insbesondere die angemessene Nachbereitung der von den Lernenden produzierten Videosequenzen von Bedeutung.

Folgende organisatorische Punkte sind bei dem Einsatz einer Filmkamera im FSU zu bedenken: die technische Ausrüstung sollte im Hinblick auf ihre Funktionstüchtigkeit und Kompatibilität vor dem Einsatz im Unterricht überprüft werden, um Pannen zu vermeiden.

Die Gruppengröße und die räumlichen Möglichkeiten sind zwei weitere Faktoren, die es $\mathrm{zu}$ berücksichtigen gilt. Kleingruppenarbeit, verbunden mit der Möglichkeit einen weiteren Raum zu benutzen, ermöglicht ein selbständiges und intensives Arbeiten der Lernenden.

Für den handlungsorientierten Gebrauch der Videokamera im FSU wird im Folgenden ein Überblick über bewährte Übungsformen und Themen gegeben. Anschließend wird die wichtige Phase der Nachbereitung der angefertigten Aufnahmen thematisiert.

\section{Übungsformen und Themen}

Der handlungsorientierte Gebrauch der Filmkamera eignet sich besonders für sog. kommunikative Übungsformen. Die folgende Auflistung enthält eine Sammlung von bewährten Übungsformen und Themen.

\section{Präsentationen}

Diese Form wird im Zusammenhang mit Film auch als talking head bezeichnet. Einzelne Lernende halten vor der Videokamera eine kleine Rede oder ein Referat zu einem Thema ihrer Wahl. Dabei können die Lernenden u. a. die Strukturierung einer Präsentation in der Fremdsprache durch den Gebrauch von Konnektoren lernen und üben. (vgl. Lonergan 1990, S. 6-10) Für Präsentationen sind geeignet: Beschreibungen (z. B. Meine Heimatstadt; Mein Heimatland; Hobbies etc.), Geschichten, Erzählungen, Erlebnisberichte, Instruktionen und Erklärungen, Spekulationen (z. B. Drei Wünsche; Wenn ich die Welt regieren würde ...; ), Appelle (z. B. Umweltschutz, Politik, Religion), Ansagen, Werbung, Lyrik, Lieder.

\section{Rollenspiele}

Diese werden durch das Entwickeln von Dialogen vorbereitet, um dann nachgespielt und auf Video aufgenommen zu werden. Rollenspiele sind besonders dafür geeignet, Alltagssituationen in der Fremdsprache nachzuspielen. Dabei sind zu berücksichtigen: Situation/Thema, Rollen, Redeabsichten/Redemittel und Arrangement. (vgl. Pauels 1995, S. 237) Rollenspiele bieten sich für folgende Bereiche an: Alltagstransaktionen (z. B. Einkäufe, Restaurants), Intentionen (z. B. jmd. überzeugen etwas zu tun, Arbeitsvorgänge erklären, ver-

\section{handeln.}

\section{Simulationen}

Die Übergänge vom Rollenspiel zur Simulation sind fließend. Bei Simulationen wird stärker als bei Rollenspielen davon ausgegangen, dass die Lernenden als sie selbst agieren. Simulationen werden als komplex bezeichnet, weil sie Diskussionen in der Fremdsprache beinhalten, „gedacht als Nachempfindung einer vorgestellten gesellschaftlichen Wirklichkeit" (Pauels 1995, S. 237). Simulationen zielen darauf ab, die Lernenden mit kommunikativen Mitteln für Diskussionen in der Fremdsprache vertraut zu machen. Die Analyse des Verlaufs und der Struktur einer fremdsprachlichen Diskussion trägt dazu bei, die Reflexion des fremdsprachlich-kommunikativen Verhaltens zu ermöglichen (vgl. ebd., S. 237). Simulationen können z. B. beinhalten: Diskussionen verschiedener Interessensgruppen und Funktionsträger, Entscheidungsgremien, Kontroversen zu angemessenen Themen.

\section{Sketche und Theaterspiele}

Die Spannbreite der Möglichkeiten für diese kommunikative Übungsform ist groß. Ausgehend von dramatischen, epischen, lyrischen oder selbst verfassten Textvorlagen können fiktive Situationen und Handlungen mit fiktiven Charakteren anlog zu Elementen verschiedener Textsorten bzw. Genres (Tragödie, Komödie, Krimi, Science-Fiction, etc.) für die Videoproduktion inszeniert oder improvisiert werden. Geeignete Textvorlagen für diese Form sind Dramen, Kurzdramen, Märchen, Parodien, Satiren oder Witze.

\section{Vertonung}

Bei der Vertonung von selbst produziertem oder vorhandenem Filmmaterial lassen sich zwei Formen unterscheiden: der OFF-Ton-Kommentar und die Synchronisation. Die Vertonung wie auch die anderen Übungsvorschläge eignen sich für die Vorbereitung größerer Vorhaben.

\section{Die Nachbereitung der Filmaufnahmen}

Filmaufnahmen ermöglichen in besonderer Weise das Schulen der Reflexion und die Anwendung metakognitiver Strategien auf das Sprechen in der Fremdsprache. Es ist zu betonen, dass die Nachbereitung der entstandenen Filmaufnahmen in einer vertrauensvollen Atmosphäre durchgeführt werden muss, um den Lernerfolg nicht zu gefährden, d. h., dass Feedbackregeln formuliert werden sollten und Lehrende aktiv zur Stärkung des Selbstvertrauens der Lernenden beitragen sollten, um die Hemmungen beim Sprechen in der Fremdsprache zu verringern. (vgl. Lonergan 1990, S. 27) Zuerst müssen die Lernenden, die gefilmt wurden, eine Chance erhalten, ihre Darbietung zu kommentieren und zu korrigieren, danach können sich Lernende, 
die nicht direkt beteiligt waren, zur Aufnahme äußern. Nach Lonergan (1990) wird ein bewährtes fremdsprachendidaktisches Analysemodell verwendet, das an die erste Stelle die Analyse der Diskursebene

wendet, das an die erste Stelle die Analyse der Diskursebene setzt, um den Erfolg des gesamten Kommunikationsereignisses einzuschätzen. Das sprachliche Register, die Angemessenheit der verwendeten sprachlichen Mittel und die non-verbalen Mittel sind weitere Ebenen einer möglichen Analyse. Erfahrungsgemäß ist die Analyse spezifischer Phrasen auf der Satzebene bis zur syntaktischen, lexikalischen und morphologischen Analyse stark lernergruppenabhängig. Videoaufnahmen eignen sich nicht für die Grammatikarbeit und sollten niemals dazu verwendet werden, einzelne Lernende, vorzuführen'. Videoaufnahmen sind hingegen in besonderem Maße dazu geeignet, phonetische Probleme beim Sprechen in der Fremdsprache zu thematisieren. Die phonetische Analyse zielt einerseits auf die Verbesserung der Suprasegmentalia (Intonation, Akzent, Rhythmus) und andererseits auf die kontrastive Analyse von Interferenzen auf der Einzellautebene ab.

Diese speziell fachdidaktischen Möglichkeiten einer Nachbereitung von Videoaufnahmen sollten in einen thematisch-kommunikativ orientierten FSU eingebettet sein und können deren kreative und kommunikative weitere Verwendung einschließen (z. B. thematische Dossiers, Klassenkorrespondenz).

\section{Filmprojekte im Fremdsprachen- unterricht}

\section{Voraussetzungen}

Die Ausgangssituation für Filmprojekte ist im schulischen Regelbetrieb und in der Erwachsenenbildung aufgrund des geringen zeitlichen Handlungsspielraums häufig ungünstig, weil zwei wichtige Voraussetzungen selten erfüllt werden: Filmprojekte erfordern zum einen intensive Lernphasen wie im Blockunterricht, in einer Projektwoche oder in einem Austauschprogramm. Zumindest für die Dreh- und Montagearbeiten sollte das Arbeiten an einem Stück möglich sein (vgl. Bufe 1993, S. 11). Filmprojekte erfordern zum anderen das Lernen anhand einer inhaltlichen Zielsetzung, was sich in einen Fremdsprachenunterricht, der eine grammatische Progression in den Mittelpunkt stellt, schwer vereinbaren lässt. Aufgrund der Komplexität von Filmprojekten in sprachlicher Hinsicht sind sie nicht für den Anfangsunterricht geeignet. Die Teilnahme an einem Filmprojekt sollte auf freiwilliger Basis erfolgen, zumindest eine Freiheit der Themenwahl, der Textsorte und der dramaturgischen Gestaltung sollte garantiert sein (vgl. Bufe 1993, S. 9).

Die Rolle der Lehrenden im Rahmen eines Videoprojektes sollte eine Beratungsfunktion erfüllen. Diese Beratungsfunktion erstreckt sich über den gesamten Produktionsprozess, daher werden Kenntnisse im Umgang mit der Videotechnik und Wissen über die Prinzipien der Filmgestaltung und unterschiedlicher Genres benötigt (vgl. Schell 1993, S. 200).

\section{Phasen und Ziele}

Zunächst werden einige übergreifende Ziele einer Filmproduktion im FSU genannt. Anschließend werden die verschiedenen Phasen einer Eigenproduktion dargestellt.

Unter fremdsprachendidaktischen Gesichtspunkt bleibt nach Bufe (1993, S. 9, 11), dessen Erfahrungen aus dem studentischen Fremdsprachenunterricht und der interkulturellen Begegnungsarbeit stammen, festzuhalten: „Der Wechsel vom familiären $O$ n zum förmlichen Off kann das Bewusstsein für unterschiedliche sprachliche Register schärfen“ (Bufe 1993, S. 9) (Hervorhebungen durch den Autor). Die intensive Arbeit an einem Filmprojekt führt zu einer Vertrautheit mit dem Sprachmaterial, vergleichbar mit gut memorierten Texten, die auch auBerhalb des Filmkontextes abgerufen werden können. Daher bietet die Filmproduktion im Vergleich zur Filmrezeption Möglichkeiten für ein besonders, ,intensives Sprachtraining“ (Bufe 1993, S. 11) (Hervorhebung durch den Autor). Die Kleingruppenarbeit im Rahmen eines Filmprojekts zielt immer auch auf die Ausbildung individueller und kooperativer Fähigkeiten ab (vgl. Alrø 1987, S. 33). Wie Bufe festhält, liegt im Unterschied zur Rezeption von audiovisuellen Medientexten der „didaktische Ort der linguistischen Problemlösung [...] bei der Videoeigenproduktion vor der Fertigstellung des Endproduktes“ (Bufe 1993, S. 10). Der Autor unterscheidet entsprechend drei Phasen: Die ,produktionsorientierte Entwicklungsphase" (Bufe 1993, S. 7) wird von der Realisierung der Dreharbeiten gefolgt. Im Anschluss wird in der „produktorientierten Auswertungsphase“, die Aufbereitung der Filmmaterialien durchgeführt (ebd.). Unter Einbeziehung verschiedener anderer Autoren und eigener Erfahrungswerte werden im Folgenden fünf erforderliche Phasen einer Eigenproduktion im FSU unterschieden.

\section{,Vorentlastung'}

Die Vorbereitung einer Eigenproduktion muss sicherstellen, dass die Lernenden über die notwendigen Teilkompetenzen verfügen. Für die angemessene technische und sprachliche Vorbereitung sind der Standard der Filmproduktion, die technischen Gegebenheiten und das Vorwissen der Lernenden zentral. Die Anwendung von Formen der visuellen Gestaltung, die auf das Konzipieren einer Filmproduktion vorbereiten, kann z. B. im Rahmen einer Unterrichtsreihe durch das Entwickeln eines Fotoromans kennen gelernt werden (Unterrichtsvorschläge und Beispiele s. Schwerdtfeger 1989). Gestaltungsprinzipien für einen Film sind immer genrespezifisch. Die Reflexion ver- 
schiedener Sendeformen und deren Charakteristika stellen eine weitere Möglichkeit dar eine Eigenproduktion vorzubereiten.

Auch sind kleinere Produktionen im handlungsorientierten Unterricht mit Video dazu geeignet, schrittweise eine größere Eigenproduktion vorzubereiten. Charge und Giblin beschreiben als Ziele der Vorbereitung:

„1. To give the participants the basic technical knowledge and vocabulary to enable them to write, film, and edit a short sequence of film fairly competently. 2. To use the medium of task-based lessons to teach the students the necessary vocabulary and language patterns to enable them to cope with the video tasks entailed in film production" (Charge; Giblin 1988, S. 283).

Die Vorbereitung sollte also sicherstellen, dass die Lernenden die grundlegenden spezifischen Gestaltungsmittel von Film kennen, die Geräte bedienen können und die erforderlichen Redemittel für eine Filmproduktion beherrschen.

\section{Themenfindung}

Für den Fremdsprachenunterricht sind interkulturelle Perspektiven bei der Themenfindung besonders interessant. Für die Themenfindung sollten ggf. Anregungen in Form von Einstiegsmaterialien gegeben werden, wie z. B. thematische Filme oder Eigenproduktionen anderer Gruppen, Zeitungsausschnitte, literarische Vorlagen (vgl. Schell 1993, S. 197).

\section{Enwicklung}

Bereits bei der Planung und Entwicklung eines Filmprojekts müssen die technischen Bedingungen berücksichtigt werden, um dann in der Realisierungsphase eine Diskrepanz zwischen den entwickelten Ideen und den technischen Möglichkeiten zu verhindern.

An die Themenfindung schließen sich folgende Schritte an: Zunächst wird der Inhalt des geplanten Videofilmes in erzählender Weise in Form einer Filmgeschichte zusammengefasst. Danach wird die Filmgeschichte im Drehbuch (und/oder Story-Board) in verschiedene Szenen zerlegt. Der organisatorische Ablauf der Dreharbeiten wird im Drehplan vermerkt wie auch Angaben über erforderliche Ausstattung. In Abhängigkeit vom Genre sind Texte zu erstellen und Proben z. B. für das Führen von Interviews zu planen.

\section{Realisierung der Dreharbeiten}

Es kann sich empfehlen während der Dreharbeiten innerhalb und zwischen den verschiedenen Kleingruppen eine Aufgabenrotation $\mathrm{zu}$ vereinbaren oder die Rollenzuweisung zu thematisieren, um einem (geschlechtsstereotypen) Spezialistentum vorzubeugen (vgl. Schell 1993, S. 198-199, Bufe 1993, S. 8). Die Gestaltung der Szenen und die Aufnahmen erfolgen anhand von Drehbuch und Drehplan. Das Anfertigen von Filmaufnahmen erfordert Sensibilität gegenüber den zu Filmenden, was den Lernenden bewusst sein sollte. Es kann sinnvoll sein innerhalb der Gruppe vorab Regeln zu vereinbaren, die ungewollte Aufnahmen untereinander ausschließen

\section{Produktorientierte Auswertung}

Sind die Dreharbeiten abgeschlossen, erfolgt die Gestaltung der Filmaufnahmen durch Montage und Schnitt sowie die Vertonung des Films oder einzelner Szenen. In Abhängigkeit von der Art des entstandenen Films ist die Abschlussphase des Projektunterrichts mit einer Form der Öffentlichkeitsorientierung verbunden. Der fertig gestellte Film kann für verschiedene Adressatenkreise zugänglich gemacht werden, z. B. durch Vorführung, durch die Integration in Intra- oder Internet-Seiten oder durch die Verwendung in einer E-Mail-Korrespondenz.

\section{Ausblick}

Lange Zeit ist im FSU die isolierte Vermittlung sprachlicher Fertigkeiten betrieben worden (Eggers 1996). Deshalb ist es notwendig, auf die inzwischen erkannte Bedeutung der Verzahnung der verschiedenen sprachlichen Fertigkeiten hinzuweisen, denn einer der Vorteile des Mediums Film ist, dass es die Integration dieser Fertigkeiten geradezu herausfordert. Seit den Kindertagen des analogen Videos sind filmpädagogische Ansätze für den FSU entwickelt und erprobt worden, seit Mitte der neunziger Jahre scheint deren Weiterentwicklung aus der fremdsprachendidaktischen Diskussion verschwunden zu sein. Gleichzeitig sind Videos mit der Entwicklung vom analogen zum digitalen Film zum gängigen Bestandteil multimedialer Anwendungen und gängiger Lehrwerke geworden.

In der Verwendung digitaler Aufnahme-, Wiedergabe- und Distributionsformen liegt Potenzial für die Wiederbelebung filmpädagogischer Arbeitsformen im FSU. Einerseits ist die handlungsorientierte Arbeit mit Film durch Digitalisierung besser zu handhaben und dadurch vereinfacht z. B. in eine E-Mail-Klassen-Korrespondenz einzubinden. Andererseits verlangt die zunehmende Medienkonvergenz, um deren Synergieeffekte auch pädagogisch nutzbar zu machen, eine Weiterentwicklung bestehender pädagogischer Ansätze hin zu integrativen und kritischen Ansätzen, wie es z. B. Mayer und Treichel (2004) in ihrem Band zu handlungsorientiertem Lernen und eLearning versuchen.

\section{Zusammenfassung}

Der Beitrag präsentiert bewährte Vorschläge für die handlungsorientierte Arbeit mit Film im Fremdsprachenunterricht. Eingangs werden die 
Geschichte und die Verwendungsgebiete von Film im Fremdsprachenunterricht skizziert. Das Entwickeln (fremdsprachiger) Medienkompetenz wird als übergeordnetes Lern-/Lehrziel betrachtet, das integraler Bestandteil der Arbeit mit Film im Fremdsprachenunterricht ist. Die Darstellung der erprobten Verwendungsweisen von handlungsorientiertem Video im Fremdsprachenunterricht wird mit einem Ausblick abgeschlossen, der vor dem Hintergrund einer zunehmenden Medienkonvergenz die Notwendigkeit für die Weiterentwicklung integrativer und kritischer Ansätze aufzeigt.

\section{Anmerkung}

Nicht zuletzt sind ggf. Rechtsvorschriften für die Filmaufnahmen zu beachten. Für die Verbreitung und öffentliche Wiedergabe von Videoproduktionen sind die wichtigsten betroffenen Rechte das allgemeine Persönlichkeitsrecht und das Recht am eigenen Bild, d. h., dass die Einwilligung der im Film abgebildeten Personen schriftlich oder auf Band vorliegen sollte. Für Innenaufnahmen muss eine Drehgenehmigung vom Inhaber des Hausrechts eingeholt werden. Wenn beabsichtigt wird, eine Videoproduktion $\mathrm{zu}$ veröffentlichen, sollte berücksichtigt werden, dass bei der Verwendung von Fremdmaterialien (Musik, Fernsehmitschnitte), die damit verbundenen Urherber- und Leistungsschutzrechte zu beachten sind. Die Verwendung geschützter Musikstücke ist grundsätzlich zu vermeiden, ggf. sollte die Hilfe von Musiker/innen oder Musikgruppen beansprucht werden.

\section{Literatur}

Alrø, Helle: Überlegungen zu einer Medienpädagogik für den Fremdsprachenunterricht Deutsch in Dänemark. In: Ehnert, Rolf; Eppeneder, Ralf (Hrsg.): Video im Fremdsprachenunterricht. Materialien zweier Fortbildungsseminare. München: Goethe-Institut 1987, 23-36.

Bufe, Wolfgang: Der Einsatz der Videokamera im interkulturellen Fremdsprachenunterricht. In: Jung, Udo O. H. (Hrsg.): Praktische Handreichung für Fremdsprachenlehrer. Frankfurt a. M.: Peter Lang 1992, 199-213.

Bufe, Wolfgang: Videogestützter Fremdsprachenunterricht: von der Medienrezeption zur Medienproduktion. In: Der fremdsprachliche Unterricht - Französisch 2/93, 7 ff.

Charge, Nick J.; Giblin, Karen: Learning English in a video studio. In: English Language Teaching Journal 42/4, 1988, $383 \mathrm{ff}$.

Eggers, Dietrich: Einführung in die Problematik des thematischen Teils (Medientheorie und Medienpraxis in Sprachlehr- und lernprozessen). In: Jahrbuch Deutsch als Fremdsprache. 7, 77; München: Iudicum Verlag 1981.

Eggers, Dietrich: Hörverstehen: Bestandsaufnahme und Perspektiven. In: Kühn, Peter (Hrsg.): Hörverstehen im Unterricht Deutsch als Fremdsprache: theoretische Fundierung und unterrichtliche Praxis. Frankfurt a. M.: Peter Lang 1996, 13-44

Fuß, Albert, 1992: Vom Dia zum Video - Entwicklungen im audiovisuellen Sprachunterricht. In: Jung, Udo O.H. (Hrsg.): Praktische Handreichung für Fremdsprachenlehrer. Frankfurt a. M.: Peter Lang, 155-163.

Kühn, Olaf 1998: Vom Sehen plus Hören zum Sprechen. Zu den Chancen des Videoeinsatzes im Französischunterricht. München: Kopaed Verlag

Lindhorst, Monika; Röllecke, Renate: Audiovisuelle Medien kompetent nutzen. In: Informationen Deutsch als Fremdsprache, 23,1, 1996, 95106

Lonergan, Jack: Video in Language Teaching. Cambridge University Press 1984

Lonergan, Jack: Making the Most of Your Video Camera. CILTR: London 1990

Mayer, Horst Otto; Treichel, Dietmar (Hrsg.): Handlungsorientiertes Lernen und eLearning. Grundlagen und Praxisbeispiele. Oldenbourg Wissenschaftsverlag 2004

Pauels, Wolfgang: Kommunikative Übungen. In: Bausch, Karl-Richard; Christ Herbert; Krumm, Hans-Jürgen (Hrsg): Handbuch Fremdsprachenunterricht. Francke: Tübingen, Basel 1995, 236-238.

Röllecke, Renate: Vom medialen Ersatzlehrer zum Diskussionsanlaß. In: Info DaF 18,4, 1991, 405-419, München: Iudicum Verlag

Schell, Fred: Aktive Medienarbeit mit Jugendlichen, Theorie und Praxis. München: Kopaed Verlag 1993

Schwerdtfeger, Inge C.: Sehen und Verstehen Arbeit mit Filmen im Unterricht Deutsch als Fremdsprache. Berlin u. München: Langenscheidt 1989

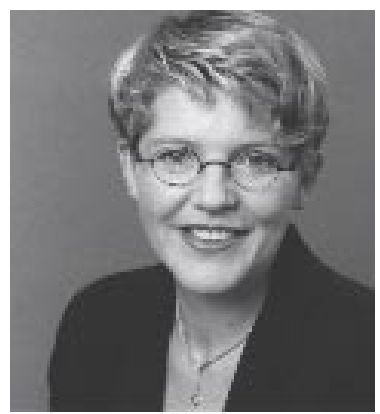

\section{Dr. phil. Marion Niehoff}

Dozentin, Lehrbeauftragte

Arbeitsschwerpunkte:

Deutsch als Fremdsprache, Fremdsprachen didaktik, Fremdsprachen in der Berufswelt, Medienpädagogik/-didaktik (eLearning, Video), qualitative Sozialforschung, Studie: „Fremdsprachenlernen mit Multimedia. Eine qualitative Untersuchung zum selbstorganisierten Lernen“, Informationen unter

http://www.niehoff.gmxhome.de 